\title{
Multifluid macroscopic approach to drying in papermaking
}

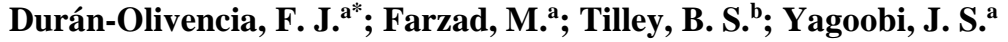

${ }^{a}$ Multi-Scale Heat Transfer Laboratory, Mechanical Engineering Department, Worcester Polytechnic Institute, Worcester, MA 01609, USA

b Department of Mathematical Sciences, Worcester Polytechnic Institute, Worcester, MA 01609, USA

*E-mail of the corresponding author: fjduranolivencia@wpi.edu

\begin{abstract}
Drying process represents one of the main energy-consuming stages for a broad variety of manufacturers. However, despite its significant impact on energy efficiency, most implementations at manufacturing level do not operate within the optimal conditions.

This work investigates the interplay among different parameters involved into the paper-drying process. To do so, we analyze both experimental and numerical results. The theoretical approach couples a non-isothermal flow, along with the heat transfer and transport of different fluids participating. Our results, experimental and numerical, show a good agreement according to the characteristic drying time scale. The results thus enable to estimate the impact of different mechanisms into drying process.
\end{abstract}

Keywords: paper-drying process; free water; bound water; papermaking. 


\section{Introduction}

Drying porous media is an experience surrounding us every single day. Food, building material, paper pulp, textiles or tissues are some examples of the engineering applications wherein the drying process has a central impact.

Porous media refers to materials presenting a pores network. In most applications, these cavities are filled with different fluids: gases and liquids. The interaction between those fluids and the solid matrix is what ultimately regulates the mass transfer concurring in this complex pores network. Indeed, understanding the complex dynamic of drying rate has been of interest for the past decades. At high saturation level, the water may be described using its bulk properties, that is called free water[1]. Although the latter represents a significant percentage of the water inside the structure (roughly $80 \%$ in most cases), drying the remaining water, physical or chemically adsorbed into the solid matrix (bound water), requires a considerable energetic effort.

Unfortunately, an exact description about bound water demands a microscopic approach for the solid-fluid interaction. Such description involves knowing the peculiarities of geometrical irregularities as well as forces at the molecular level between walls and fluid, which turns the problem into a challenge both theoretical and numerical. Nonetheless, in most practical cases, a so accurate insight is unnecessary or leads to an impossible undertaking.

To tackle this multi-scale scenario, we investigate the drying process on paper using the porous media theory. We followed, therefore, a macroscopic approach which provides an intuitive description averaging all minutiae at the microscopic level. We turn in this way the micro-scale effects into volume and surface terms in the governing equations at macroscale[2]-[4].

This paper analyze a real case in which a non-isothermal flow is addressed to the porous medium, initially wholly saturated. We explore thus the interplay among mass and energy transfer, pressure distribution, or the evolution of evaporation front among other parameters. To do so, we combine a numerical approach with experimental results. Our results enable to clarify the transition between free and bound water, elucidating the role played by the main control parameters during the experiment.

Section 2 presents the problem formulation, discussing the physics and assumptions considered in the macroscopic approximation. Section 3 details the numerical and experimental results. Finally, Section 4 brings together the leading conclusions, highlighting potential mechanisms to investigate in future works to incorporate the bound water effect at low saturation level.

\section{Problem formulation}

In this section, we motivate the macroscopic formulation of the porous medium and its interaction with the flow passing it through as well as the heat transfer induced. The first paragraph is dedicated to the geometrical setting since that helps to bear in mind an intuitive workflow to formulate the corresponding governing equations later.

\subsection{Geometrical setting}

Fig. 1 shows a schematic sketch about the geometrical setup modeled in this work. As the 


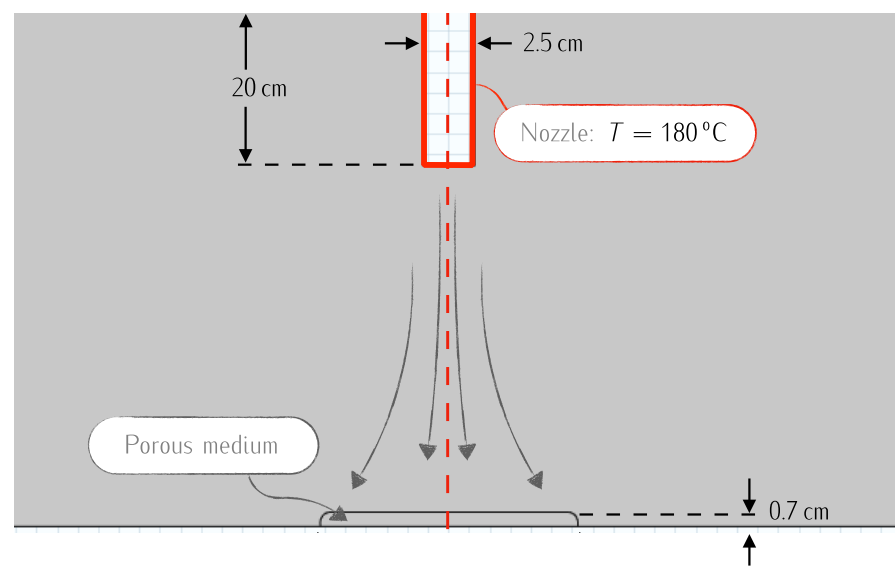

Fig. 1. Schematic sketch of the setting investigated in this work.

figure illustrates, we simulate the problem in a two-dimensional case. For low Reynolds numbers, that is a reasonable assumption that trims the computational effort considerably.

\subsection{Governing equations: A miscible fluids formulation}

The macroscopic approach comes up averaging over a representative elementary volume[5]. At this high level, there is no trace of surface topology at micro-scale, and the porous medium is then reduced to a continuum view. Solid-fluid interaction is thus modelled via a distributed energy loss induced by the flow going through the solid matrix.

Hence, at such high level, the flow field for the gas phase (dry air and water vapor) is governed by a single Navier-Stokes equation (NSE)[6]. Indeed, inside the porous medium, the energy losses induced by the solid matrix require some additional considerations. Those are, essentially, concerning on the way the solid matrix modulates different contributions in the NSE, turning eventually this into a Brinkman equation (BE)[7]. Thus, outside the porous medium, the gas-phase flow is described by a non-isothermal NSE:

$$
\begin{gathered}
\frac{\partial \rho_{g}}{\partial t}+\nabla \cdot\left[\rho_{g} \mathbf{u}_{g}\right]=0 \\
\rho_{g}\left[\frac{\partial \mathbf{u}_{g}}{\partial t}+\mathbf{u}_{g} \cdot \nabla \mathbf{u}_{g}\right]=\nabla \cdot\left[-p \mathbf{I}+\mu_{g}\left(\nabla \mathbf{u}+\nabla \mathbf{u}_{g}^{\mathrm{T}}\right)-\frac{2}{3} \mu_{g}(\nabla \cdot \mathbf{u})\right],
\end{gathered}
$$

where $\rho_{g}$ and $\mu_{g}$ are air density and dynamic viscosity, respectively. Inside the porous structure, however, Equation (1) must be modified considering the drag induced by the solid matrix, what eventually results in the $\mathrm{BE}[3]$

$$
\left(\frac{\rho_{g}}{\varepsilon}\right)\left[\frac{\partial \mathbf{u}_{g}}{\partial t}+\mathbf{u}_{g} \cdot \nabla \mathbf{u}_{g}\right]=-\nabla p+\mu_{g} \tau \nabla^{2} \mathbf{u}_{g}-\frac{\mu_{g}}{k} \mathbf{u}_{g}-\frac{c}{k^{1 / 2}} \rho_{g}\left|\mathbf{u}_{g}\right| \mathbf{u}_{g}
$$


where the last two terms of the RHS represent Darcy[8] and Forscheimer[9], [10] resistances, respectively. The coefficients $\varepsilon, k_{g}$ and $\tau$ are the porosity, permeability of the gas phase, and tortuosity, respectively. The coefficient $c$ comes from an experimental fitting; we consider the experiments carried out by Hoang et al.[11] which are an example of such empirical relationship between $c$ and $k$.

Water vapor velocity field may be described in terms of $\mathbf{u}_{g}$, including the diffusion of vapor into the dry air (binary diffusion, Bird et al.[12])

$$
\mathbf{u}_{v}=\frac{\mathbf{u}_{g}}{S_{g} \varepsilon}-\frac{M_{a} D_{e}}{M_{\nu} \rho_{v}} \nabla \rho_{\nu},
$$

where an effective porosity $\left(S_{g} \varepsilon\right)$ and diffusivity $\left(D_{e}\right)$ are considered. The first one according to the saturation level, whereas the effective diffusivity depends on the local temperature, porosity, and tortuosity[13]

$$
D_{e}=D_{\nu} \varepsilon^{4 / 3} S_{g}^{10 / 3}
$$

where $D_{v}$ is the bulk diffusivity of the water vapor in the air.

Liquid phase (water), conversely, not require such a sophisticated formulation to compute its velocity field. Water inside the porous media reaches velocities much smaller than the gas phase. This fact enables to describe the water velocity field using a Darcy's law, depending solely on the pressure gradient induced into the gas phase dynamic

$$
\mathbf{u}_{l}=-\frac{k_{l}}{\mu_{l} S_{l} \varepsilon} \nabla p,
$$

where $S_{l} \varepsilon$ reflects an effective porosity as a function of local saturation level.

The lack of concrete pores, conceptually, leads to the impossibility to track the interaction between two phases concurring within the porous medium. Essentially, because there is no way to define an interface, and consequently the interfacial energy stored. Even though, mechanisms such as capillary or evaporation may be included in the transport of different phases considering their average contribution over the REV. Phases are thus represented as miscible fluids; which is entirely consistent with the mean-field character of REV approximation. Transport of liquid and gas phases are formulated then as a conventional balance equation:

$$
\begin{gathered}
\frac{\partial c_{l}}{\partial t}+\mathbf{u}_{l} \cdot \nabla c_{l}-D_{\text {cap }} \nabla^{2} c_{l}=-\dot{m}_{\text {evap }} \\
\frac{\partial c_{v}}{\partial t}+\mathbf{u}_{v} \cdot \nabla c_{v}-D_{e}\left(\frac{M_{a}}{M_{g}}\right) \nabla^{2} c_{v}=\dot{m}_{\text {evap }}
\end{gathered}
$$

where $D_{\text {cap }}$ represents the diffussivity induced by the capillary effects[3], and $\dot{m}_{\text {evap }}$ is the mass flux evaporating

$$
\dot{m}_{\text {evap }}=v_{\text {evap }}\left(a_{\mathrm{w}} c_{\mathrm{sat}}-c_{v}\right) \text {. }
$$

The coefficient $v_{\text {evap }}\left[\mathrm{s}^{-1}\right]$ accounts the evaporation rate, whereas $a_{\mathrm{w}}$ represents the water activity[4]; $c_{v}$ is the concentration of water vapor at a given time; and $c_{\text {sat }}$ is the saturation 
concentration

$$
c_{\mathrm{sat}}=\frac{p_{\mathrm{sat}}(T)}{R T},
$$

where $p_{\text {sat }}$ is the saturation pressure, and $R$ is the gas constant.

Nonetheless capillary, water activity, or intrinsic porous properties depend overall on the water saturation level[4]. Those levels are, however, closely related to the corresponding concentration via the relationship:

$$
S_{l}=\frac{c_{l} M_{l}}{\rho_{l} \varepsilon}
$$

which, ultimately, means the ratio of mass of a given species in an elementary volume over the total. On the other hand, given that phases are connected each other in an elementary volume via conservation of mass, the gas saturation level turns out to be:

$$
S_{g}=1-S_{l}
$$

Saturation variables alleviate the heat transfer problem inside the solid structure, reducing it to the general energy balance for a single phase flow;

$$
\rho_{m} C_{p, m}\left[\frac{\partial T}{\partial t}+\mathbf{u}_{m} \cdot \nabla T\right]=\nabla \cdot\left(k_{m} \nabla T\right)-\mathscr{L}_{\text {evap }} \dot{m}_{\text {evap }}
$$

where the subscript $\dot{m}_{\text {evap }}$ indicates the average thermal properties, which results from a weighted average between the two phases coexisting within the porous structure

$$
\begin{aligned}
\rho_{m} & =S_{g} \rho_{g}+S_{l} \rho_{l}, \\
C_{p, m} & =\frac{C_{p, g} S_{g} \rho_{g}+C_{p, l} S_{l} \rho_{l}}{\rho_{m}} \\
k_{m} & =S_{g} k_{g}+S_{l} k_{l} \\
\mathbf{u}_{m} & =\frac{\Gamma_{g} C_{p, g}+\Gamma_{l} C_{p, l}}{\rho_{m} C_{p, m}}
\end{aligned}
$$

where

$$
\Gamma_{l, g}=\rho_{l, g} \mathbf{u}_{l, g}
$$

is the flux of each phase.

Finally, according to the modeling detailed in this section, the water dynamic inside the porous structure is described as a combination of different mechanisms interconnected with each others. Hence, free and bound water concepts are not considered as discrete states within the continuum approach, but rather as asymptotic modes induced by a combination of mechanisms involve in drying process.

\subsection{Implicit assumptions and limitations}

The theoretical model above mentioned connects micro- and macro- scales via water activity and solid matrix properties. There are two critical points, however, assumed in such 
continuum description:

- Water activity. Although the theoretical model is part of a macroscopic framework, water activity (Eq. (7)) brings in the microscopic dynamic of water via sorption isotherms. We followed the IUPAC recommendations[14], which mainly divide the physisorption mechanisms into five classes according to the original classification carried out by Brunaer et al.[15]. In particular, we implemented the type I (Langmuir isotherm), since that is commonly exhibited in micropores.

- Average properties. Porous media properties used in this work were chosen from both theoretical and experimental relationships for different porous media including foods[4].

\section{Results and discussion}

This section itemizes the major findings derived from the theoretical model introduced in the previous section.

\subsection{Drying curve}

Fig. 2 (left) shows the numerical drying curve. The evolution of moisture content exhibits, mostly, two different areas: a linear region, which is linked in the literature to free water; and another nonlinear region at low saturation levels associated with bound water wherein surface effects become dominant.

As we mentioned above, the theoretical model presented in this work is not aimed at differentiating between free and bound water, at least in the conventional manner. Mainly, because these concepts revolve around discrete states, which is hardly fit with the continuum framework proposed. Water activity and the corresponding sorption isotherm are used then
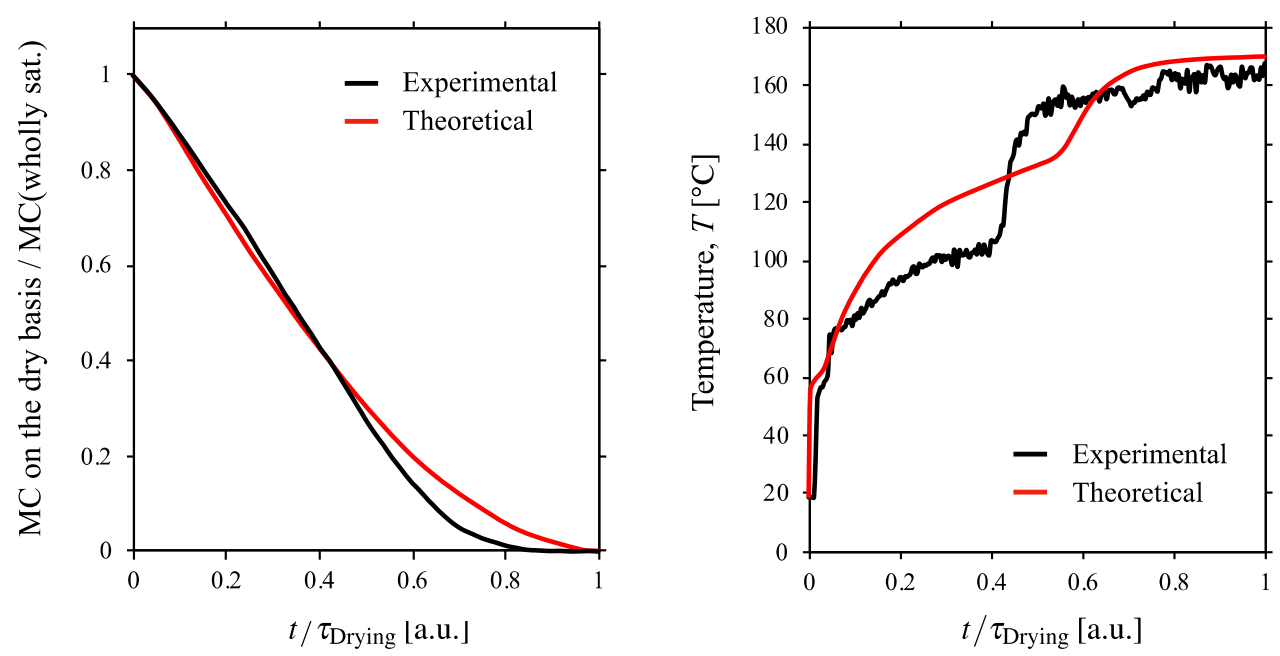

Fig. 2. (Left) Drying curve. (Right) Temperature evolution at surface center. Both graphs are

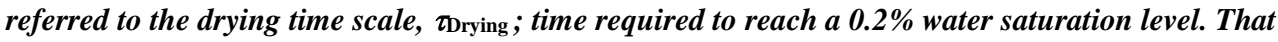
enables a qualitative comparision between experimental and theoretical results. Experimental curves were obtained according to the setup detailed by Farzad et al. [16]. 
as the natural way to connect micro- and macro-scales concurring along the transient. Therefore, the nonlinear region appears as a combination of different factors involving not only fluid-wall interaction at micro-scale via water activity, but also the evolution of others macroscopic magnitudes as the pressure and temperature distributions.

The good agreement (qualitatively) between numerical and experimental curves supports the theoretical insight proposed in this work. Such validation enables to explore the role of each macroscopic control parameters in the sorption process, and thereby in the drying dynamic.

\subsection{Temperature evolution at surface center}

Fig. 2 (right) displays another characteristic response throughout the drying process evolution: temperature evolution at the surface center of the sample.

Surface center is the point at which the flow is addressed (Fig. 1). The flow outside porous structure quickly reaches a steady situation. As the curve details (Fig. 2-right), the two-step profile reveals the dependency with other factors such as the evolution of evaporation front. Otherwise, the combination of convection and conduction mechanisms would lead to a single step curve.

\section{Conclusions}

Recent work about the drying dynamic inside porous media provides a continuum theoretical framework to deal with multiple scales concurring during the drying process. Free and bound water are posed as asymptotic water modes inside the solid structure rather than two different and discrete states. Our results are in good agreement (qualitatively) with their experimental counterparts. Drying curve exhibits not only the linear part usually assigned to free water, but also nonlinearities associated with bound water at low saturation levels. In its turn, the temperature evolution at the surface center of the sample presents the two-step profile observed experimentally. On the other hand, the pressure and temperature distributions inside the solid structure unveil the way the evaporation front advances through the solid matrix. The nonlinear advancement of the evaporation front through the porous structure pushes the water to the edge of the domain where the heat transfer drops considerably

Nevertheless, these results must be analyzed with extreme care since we assumed two critical points. First, we considered a Langmuir adsorption isotherm as the sorption dynamics modulating the water activity inside the solid structure. While it is true that it is commonly used in micropores, there is no enough experimental evidences to support this assumption in the case we worked with. For instance, capillary condensation is a potential adsorption isotherm to be considered, since it explains the way alternating wide and narrow parts of pores may enhance evaporation or condensation processes. On the other hand, solid matrix properties were considered as an average based on a bunch of theoretical and experimental relationships including different porous media.

Therefore, given that the theoretical framework presented in this work connects the microand macro- scales via porous medium properties and the adsorption isotherm, a more complete description of these two factors is required. They do are currently under a more indepth investigation in our laboratory both experimental and theoretically.

\section{Acknowledgements}

This study was financially supported by the Center for Advanced Research in Drying 
(CARD), a US National Science Foundation Industry University Cooperative Research Center. CARD is located at Worcester Polytechnic Institute and the University of Illinois at Urbana-Champaign (co-site).

\section{References}

[1] P. A. Vesilind, "The Role of Water in Sludge Dewatering," Water Environment Research, vol. 66, no. 1, pp. 4-11, 1994.

[2] M. C. Asensio, "Transport phenomena during drying of deformable, hygroscopic porous media: Fundamentals and applications,” PhD Thesis, 2000.

[3] A. K. Datta, "Porous media approaches to studying simultaneous heat and mass transfer in food processes. I: Problem formulations,” Journal of Food Engineering, vol. 80, no. 1, pp. 80-95, 2007.

[4] A. K. Datta, "Porous media approaches to studying simultaneous heat and mass transfer in food processes. II: Property data and representative results," Journal of Food Engineering, vol. 80, no. 1, pp. 96-110, 2007.

[5] J. Bear, Dynamics of flow in porous media. 1972.

[6] G. K. Batchelor, An Introduction To Fluid Dynamics, Cambridge University Press. 2002.

[7] H. C. Brinkman, "A calculation of the viscous force exerted by a flowing fluid on a dense swarm of particles,” Flow, Turbulence and Combustion, vol. 1, no. 1, p. 27, Dec. 1949.

[8] H. Darcy, Les fontaines publiques de la ville de Dijon: exposition et application ... Victor Dalmont, 1856.

[9] J. É. J. Dupuit, Études théoriques et pratiques sur le mouvement des eaux dans les canaux découverts et à travers les terrains perméables: avec des considérations relatives au régime des grandes eaux, au débouché à leur donner, et à la marche des alluvions dans les rivières à fond mobile. Dunod, 1863.

[10] S. Whitaker, "The Forchheimer equation: A theoretical development," Transport in Porous Media, vol. 25, no. 1, pp. 27-61, Oct. 1996.

[11] M. Hoang, P. Verboven, M. Baelmans, and B. Nicolaï, “A continuum model for airflow, heat and mass transfer in bulk of chicory roots," Transactions of the ASAE, vol. 46, no. 6, p. 1603, 2003.

[12] R. B. Bird, W. E. Stewart, and E. N. Lightfoot, Transport Phenomena, 2nd ed. New York: Wiley, 2002.

[13] R. Millington and J. Quirk, "Permeability of porous solids," Transactions of the Faraday Society, vol. 57, pp. 1200-1207, 1961.

[14] M. Thommes et al., "Physisorption of gases, with special reference to the evaluation of surface area and pore size distribution (IUPAC Technical Report)," Pure and Applied Chemistry, 2015.

[15] S. Brunauer, L. S. Deming, W. E. Deming, and E. Teller, "On a theory of the van der Waals adsorption of gases,” Journal of the American Chemical society, vol. 62, no. 7, pp. 1723-1732, 1940.

[16] M. Farzad, M. Yang, J. S. Yagoobi, and B. S. Tilley, "Drying of Moist Food Snacks With Innovative Slot Jet Reattachment Nozzle,” in International Drying Symposium, Valencia, Spain, 2018. 